\title{
Sub-4-micron full-field optical coherence tomography on a budget
}

\author{
HARI NANDAKUMAR*(D), ADITHYA KOKKODU SUBRAMANIA and \\ SHAILESH SRIVASTAVA
}

Department of Physics, Sri Sathya Sai Institute of Higher Learning, Prasanthi Nilayam, Anantapur 515134, India e-mail: hari@ radiosai.org

MS received 26 July 2017; revised 22 October 2017; accepted 10 November 2017; published online 8 June 2018

\begin{abstract}
We evaluate the construction and performance of an ultra-low-cost full-field optical coherence tomography (FF-OCT) instrument, also known as an optical coherence microscope. Although the cost of construction of the instrument from off-the-shelf parts is at least ten times lower than those of commercial products, sub-4-micron axial and lateral resolutions are obtained, albeit at the cost of higher acquisition times. Standard test samples are imaged and the performance of the instrument is evaluated. The device is found to be useful in measuring length, dispersion and group refractive index as well. Suggestions for bettering performance are discussed.
\end{abstract}

Keywords. Optical coherence tomography; coherence imaging; interferometric imaging.

\section{Introduction}

Optical coherence tomography (OCT) is analogous to ultrasonography or ultrasound scanning, commonly used in medicine [1], in the sense that it provides a three-dimensional view inside a sample based on reflection of a probe beam. In the case of ultrasonography, the probe beam is ultrasonic acoustic waves, while in the case of OCT, the probe consists of photons.

OCT relies on the coherence length of the illumination to provide axial resolution. Broader source spectra result in shorter coherence lengths and hence shorter axial resolutions of the tomogram. The lateral resolution is determined by confocal considerations, similar to a microscope. If the source spectrum is assumed to be Gaussian-shaped, the axial and lateral resolutions are, respectively [2, 3],

$$
\Delta z=\frac{2 \ln 2}{\pi} \frac{\lambda^{2}}{\Delta \lambda}
$$

and

$$
\Delta \mathrm{r}=\frac{4 \lambda f}{\pi D}
$$

where $\lambda$ is the centre wavelength of the illumination and $\Delta \lambda$ is the full-width at half-maximum (FWHM) of the spectral bandwidth of the illumination, $f$ and $D$ being the focal length and aperture size of the objective lens, respectively.

The basic structure of an OCT device consists of an interferometer as shown in figure 1. The light source is

*For correspondence chosen to have a large spectral bandwidth, and hence low temporal coherence. Interference occurs at the detector for those scattering points in the sample whose reflected light travel the same optical path length as in the reference arm. This interference signal is acquired and processed to create the tomogram of the sample.

The first OCT studies were done in ophthalmology $[4,5]$ and in the following decades, OCT has become a widely used tool in that field. Additional applications have been explored in cardiology with intravascular imaging, dentistry, pathology and dermatology. Non-medical uses of OCT in non-destructive materials testing, analysis of artwork and many other fields are ongoing research topics [2].

One of the reasons holding back OCT from widespread adoption in many fields is the relatively high cost of the instrument. The purchase costs of commonly used commercial devices run to five figures in dollars [6]. Many researchers are currently at work to reduce the cost of OCT devices to encourage wider use of this technology [7, 8]. We attempt to tackle this problem from a different perspective, using off-the-shelf components commonly available in optics laboratories. We built a bench-top FF-OCT for which we had to spend less than INR 25,000 (US\$350) on the components described in the next section, excluding costs of uncoated lenses, lens-holders and the like. Such a cost-effective instrument could be a useful tool for cashstrapped academic laboratories. We could use our FF-OCT in two modes - one being a wide field-of-view configuration without microscope objectives and the other with high lateral resolution, using $10 \times$ objectives. We were also able to make accurate measurements of material characteristics using this device. 


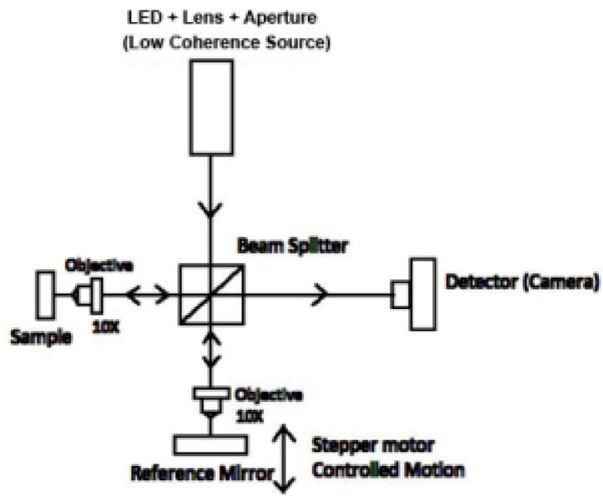

(a)

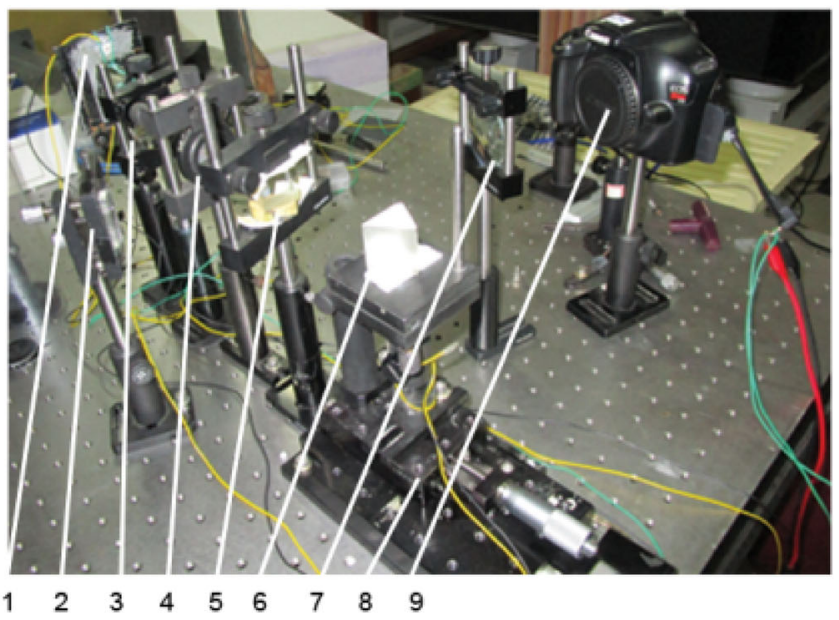

(b)

Figure 1. Full-field OCT in two modes - with and without microscope objectives: (a) schematic of the system with microscope objectives and (b) image of our set-up without microscope objectives. Labelled components are as follows: 1, LED; 2, sample; 3, lens; 4, aperture; 5, cubic beam splitter; 6 , reference mirror (front surface of a prism); 7, lens; 8 , motorized translation stage; 9 , camera.

\section{Evaluation of components}

Our approach was to implement full-field optical coherence tomography (FF-OCT), or optical coherence microscopy (OCM) [9-12] using a time domain (TD) set-up - this configuration avoids expensive galvo-mirror scanners $[3-5,13]$ and swept sources [14-16]. The use of a motorized translation stage and LED sources instead of more expensive super-luminescent diode (SLED) or halogen sources was a key driver in our cost-cutting.

Using a pre-owned entry-level DSLR camera (Canon 1100D) for image capture allowed us to have high pixel counts (12.2 megapixels) at 14-bit pixel depths [17] for just INR 7,000 (\$110). The RAW images acquired by the camera were converted to 16-bit tiff files with a linear response using DCRAW [18, 19]. Tests were done to confirm linear response of pixel values with intensity. CMOS pixel noise was a limiting factor for our camera's dynamic range. Even with the minimum gain setting of ISO 100 , averaging of 10 frames per acquisition was found to be necessary to improve signal to noise ratio (SNR) to $>40$ dB. Tests with white LED sources that had non-Gaussian spectral shapes showed us that out of the RGB values of each pixel, the $G$ and $B$ pixel values had much lower SNR. Hence, for the white LED OCT readings, only the $R$ values have been used. This also meant that the actual spectral bandwidth imaged by our device is a convolution of the source spectrum with the spectral response of the red filter of the camera sensor. This degrades the axial resolution to the experimentally measured value of approximately $3.8 \mu \mathrm{m}$ FWHM for the axial point spread function (PSF). The lateral resolution was theoretically determined to be $1.8 \mu \mathrm{m}$. We could experimentally show the lateral resolution to be better than $4 \mu \mathrm{m}$, limited by the finest calibrated image we had available, i.e., USAF target group 7. The IR filter built into the camera sensor also limited our observations to the visible spectrum. The lateral and axial resolutions experimentally obtained are pictured in figure 2 .

A single 3-W single-emitter LED source was used in each set of readings to achieve suitable illumination flux. The sources were driven at less than half their rated power $-0.25 \mathrm{~A}$ with voltage drops ranging from 2.1 to $3.2 \mathrm{~V}$ for the various LEDs - with standard lab power supplies. A pinhole placed after the light-gathering lens provided spatial filtering as well as intensity control. Intensity levels in the sample arm before the microscope objective were approximately $100 \mu \mathrm{W} / \mathrm{cm}^{2}$, as measured with an optical power meter (Newport 1830C).

Translation stages were added to each interferometer arm to aid in achieving zero path-length difference between the two interferometer arms. Micro-stepping of the motorized translation stage was carried out using a low-cost microcontroller (Arduino Uno clone), utilizing a locally sourced INR 3,300 (US\$50) micro-stepping driver (Bholanath BH MSD 2A). Though the driver allowed micro-stepping up to $39 \mathrm{~nm}$ micro-steps $(1 / 25600$ of a rotation, with $1 \mathrm{~mm}$ translation stage pitch), most of our experiments were done with micro-steps of $1.25 \mu \mathrm{m}$ (1/800 rotation). This stepping range falls well within the coherence length of our sources, and allows for quicker translations over longer optical path lengths.

Standard $10 \times$ microscope objectives, uncoated lenses and an uncoated cubic beam-splitter were used in the OCT assembly. The beam-splitter was placed at a small angle to the beams to avoid multiple back reflections from the 


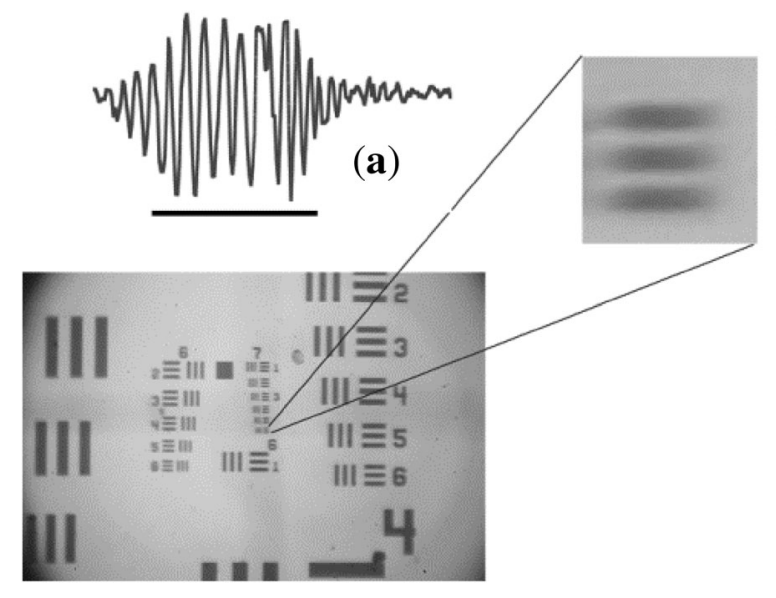

(b)

Figure 2. (a) Measured axial PSF of FWHM $3.8 \mu \mathrm{m}$ and (b) USAF target's group 7 imaged with our FF-OCT with $10 \times$ objectives showing better than $4 \mu \mathrm{m}$ lateral resolution.

beam-splitter surfaces. The reference arm had an uncoated prism to provide $4 \%$ Fresnel reflection.

\section{System assembly and experimental procedure}

Samples were chosen with well-defined upper-surface reflections for initial zero path length difference alignment. Illumination with LEDs used modified Kohler illumination, with one lens to collect the light and another to focus it onto the sample after spatially cleaning the beam through an aperture. When microscope objectives were used, the focus of the sample objective was adjusted to around half the depth of the region to be imaged, to minimize defocus due to the limited Rayleigh range of the focused beam. The operational procedure followed, in the case of sample in air, was the following:

1. Adjust the sample position by direct viewing on the camera for imaging the region of interest.

2. Move the reference arm to make its length less than the sample arm length for the top reflection of the sample, but within $1 \mathrm{~mm}$ of the sample arm length.

3. Illuminate the interferometer with a red diode laser and adjust tilt of sample to obtain coarse fringes at the camera image viewed on PC screen.

4. Illuminate the interferometer with a red LED, and move the reference arm in steps of $10 \mu \mathrm{m}$ until the zero path length position was obtained, where fringes are again seen at the camera. The red LED had a coherence length of around $20 \mu \mathrm{m}$, so the fringes were visible over a couple of least counts of the $10 \mu \mathrm{m}$ least count micrometer translation stage.

5. Illuminate the interferometer with a white LED.

6. Using an Arduino Uno to control the stepper motor and camera, move the motorized stage in micro-steps of
$1.25 \mu \mathrm{m}$, acquiring 10 images at each step, until the entire depth of region of interest has been covered.

7. Transfer the acquired images to a PC for postprocessing.

Front-surface specular reflections, though useful for initial alignment, are deleterious to our measurement technique, since our camera dynamic range is limited and we do not use balanced detection. Hence, water-immersed samples were used for imaging samples with lower scattering coefficients. The data acquisition procedure was similar to that described earlier, but needed an extra step for compensating the unequal dispersion caused by the water, present in only one arm. Dispersion-compensating glass slabs were introduced into the reference arm, and experimentally adjusted by adding and removing thin glass cover slips.

The main signal processing step in TD-OCT consists in separating the interference signal from the background. The total intensity falling on the camera is

$$
I_{\mathrm{t}}=I_{1}+I_{2}+2 I_{1} I_{2} \cos (\phi)
$$

$I_{1}$ and $I_{2}$ are the intensities of the light coming, respectively, from the reference arm and the sample arm, and the last term consists of the interference term. To subtract the background from the total signal, we used a commonly used method $[11,20]$ of subtracting adjacent frames from each other. This effectively subtracts out the background provided the phase difference between the two arms in adjacent frames is not an integral multiple of $2 \pi$.

Post-processing consisted of the following steps:

1. Conversion of the RAW images to linear TIFF images using DCRAW [18, 19].

2. Averaging of the images acquired at each step of the translation stage. This and the following image processing steps were done using Matlab ${ }^{\circledR}$ compatible GNU Octave [21].

3. Background subtraction by subtracting each step's averaged image from the previous one.

4. Reshaping the background subtracted stack of images into voxels of 3D scatterer intensity data.

5. Representing the voxels in A-scan, B-scan or C-scan form, the latter by volume rendering in Blender $[2,22,23]$.

\section{Results}

Some of our observations with this instrument include the following. The sample thickness measurements have a resolution limit of $1.25 \mu \mathrm{m}$ unless mentioned otherwise. The bulk sample thickness measurements were validated using two separate micrometer screw-gauges of least count $10 \mu \mathrm{m}$. The stepper motor translation stage measurements 
were corroborated with a micro-meter translation stage, again of least count $10 \mu \mathrm{m}$.

\subsection{PDMS micro-channels}

PDMS micro-channels of $100 \mu \mathrm{m}$ thickness were imaged in air, without microscope objectives, and the coverslip above the channel was experimentally found to be $150 \mu \mathrm{m}$ thick. Figure 3 presents a perspective view of the 3D tomographic rendering.

\subsection{Coin lettering}

A two-rupee coin was imaged as in figure 4, and the lettering was found to be $34 \mu \mathrm{m}$ thick.

\subsection{Optical fibre}

A single-mode optical fibre with its coating removed was imaged in air as well as water, as shown in figure 5. The top and bottom reflections of the fibre are obtained. The fibre diameter was found to be $135 \mu \mathrm{m}$.

\subsection{Adhesive tape}

A stack of ten pieces of transparent adhesive tape (sellotape) was imaged in water. The thickness of each layer of tape was found to vary between 23 and $36 \mu \mathrm{m}$. An edge-on view of the $3 \mathrm{D}$ reconstruction and an en-face view are shown in figure 6.

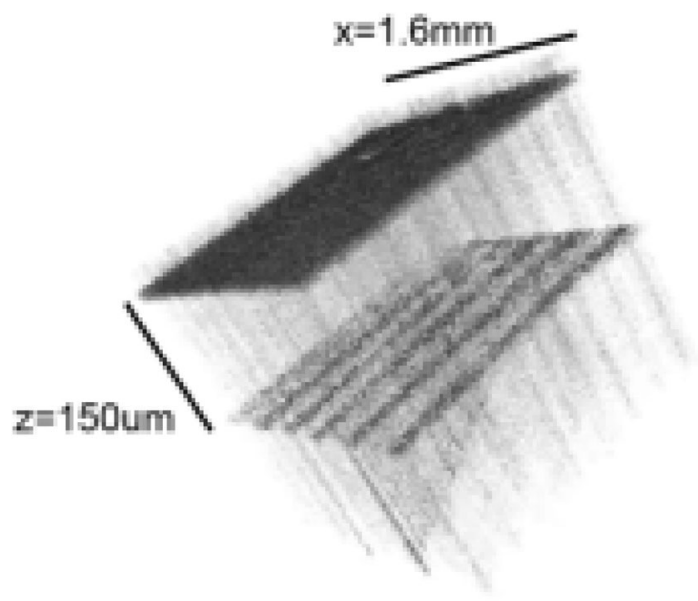

Figure 3. Tomogram of PDMS micro-channels - lateral and axial scale bars are $1.6 \mathrm{~mm}$ and $150 \mu \mathrm{m}$, respectively. (a)
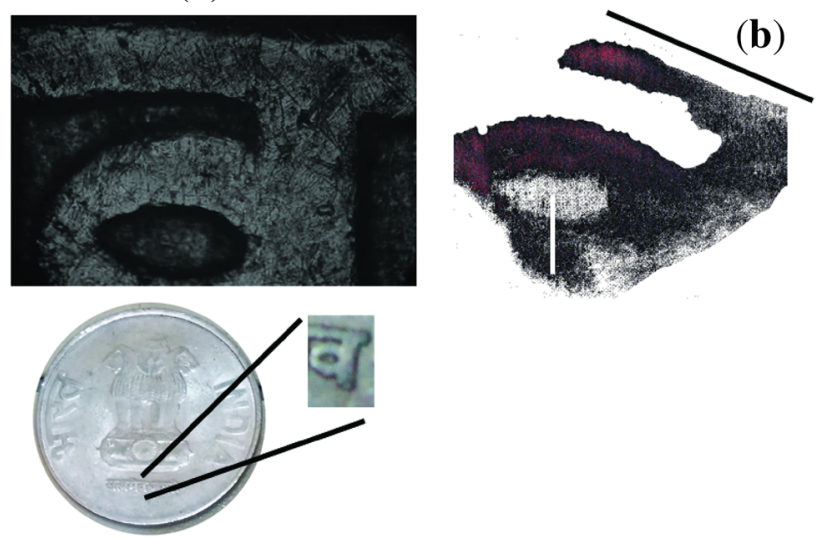

Figure 4. Image of coin and part of it imaged as a tomogram: (a) an en-face view and (b) a perspective view - white scale bar (depth) is $34 \mu \mathrm{m}$ and black scale bar (width) is $500 \mu \mathrm{m}$.

\subsection{Onion skin}

A piece of onion skin was imaged and its $3 \mathrm{D}$ reconstruction was constructed. En-face and edge-on views of the 3D reconstruction are shown in figure 7.

\subsection{Group velocity dispersion}

In the process of dispersion correction for the water immersed imaging, a measurement of the group velocity dispersion (GVD) parameter of water was performed at the red LED wavelength of $625 \mathrm{~nm}$. The water column in the working distance of the microscope objective was measured to be $4.0 \mathrm{~mm}$ long. The FWHM of the axial spread of the interference fringes from the specular reflection of a glass plate without water was measured to be $3.90 \mu \mathrm{m}$ and this was spread out to $8.97 \mu \mathrm{m}$ with the water column present. These measurements were performed with a step size of $78 \mathrm{~nm}$. The standard deviation of the axial signal spread in air is given by [24]

$$
\sigma_{\tau}=\frac{\sqrt{2 \ln 2}}{2 \pi c} \frac{\lambda_{0}^{2}}{\Delta \lambda} .
$$

The dispersed spread is given by the relation [24]

$$
\sigma_{\tau}^{\prime}=\sigma_{\tau} \sqrt{1+\left(\frac{\tau_{\mathrm{d}}}{\sigma_{\tau}}\right)^{4}}
$$

where $\tau_{\mathrm{d}}$ is the GVD time constant. Filling in our measured values $\lambda_{0}=625 \mathrm{~nm}, \Delta \lambda=20 \mathrm{~nm}$ and $\sigma^{\prime} / \sigma=2.30$, we get $\tau_{\mathrm{d}}=17.6$ fs from Eqs. (4) and (5). Using the relation [24]

$$
\tau_{\mathrm{d}}^{2}=2 l_{\mathrm{d}} \beta_{2}
$$

for our dispersive length $1_{d}=4.0 \mathrm{~mm}$, we calculate the GVD parameter $\beta_{2}$ to be $38.5 \mathrm{fs}^{2} / \mathrm{mm}$ with an $8 \%$ relative error. This agrees well with previously published data [25]. 


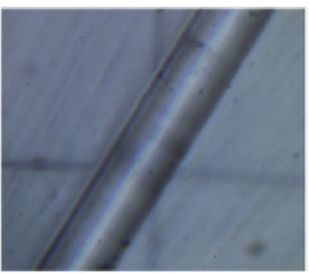

(a)

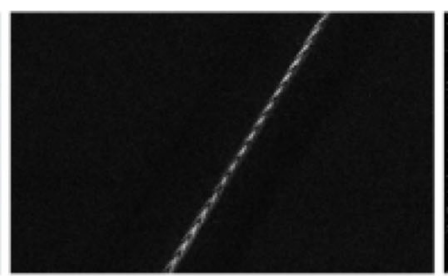

(b)

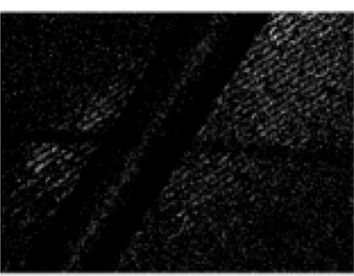

(c)

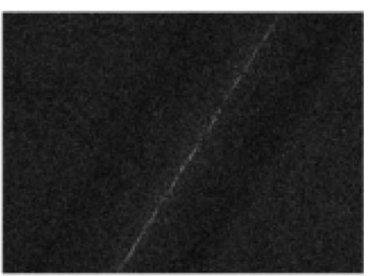

(d)

Figure 5. (a) En-face view of optical fibre. (b) Background subtracted view of top layer interference fringes. (c) Glass plate below the fibre shows up after $135 \mu \mathrm{m}$ of reference arm movement. (d) Background subtracted view of bottom layer interference fringes. The reference mirror moved by $198.75 \mu \mathrm{m}$ between images (b) and (d), which is the optical path length for $135 \mu \mathrm{m}$ of glass of $n=1.472$.

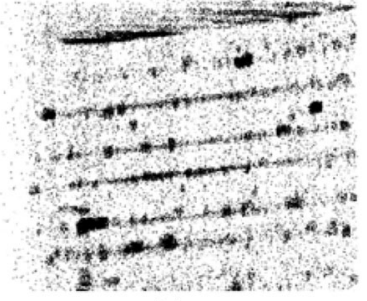

(a)

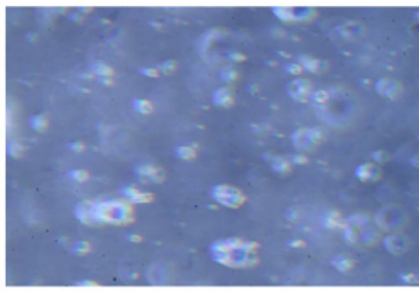

(b)
Figure 6. Sellotape stack - the blobs seen in the edge-on view of layers of tape in (a) are bubbles between the tape layers as seen in en-face image (b).

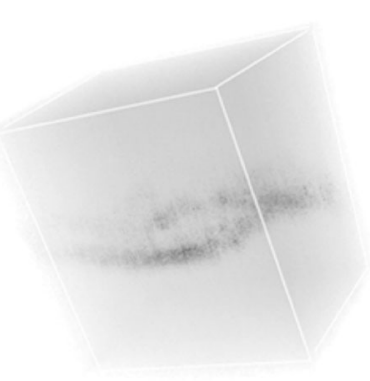

(a)

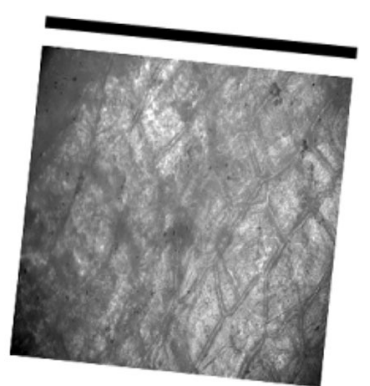

(b)
Figure 7. Onion skin: (a) edge-on view of 3D rendered data cube and (b) en-face view of onion skin, with black scale bar of $500 \mu \mathrm{m}$.

\subsection{Group refractive index}

Group refractive index measurement was performed by finding optical path length of a cuvette's inner volume first in air, and then with samples present [26]. De-ionized water and $98 \%$ pure diethyl ether solvent were the samples chosen, and readings were taken at centre wavelengths of $520 \mathrm{~nm}$ using a green LED source; the group indices were found to be 1.356 and 1.374 , respectively, with a relative error of $0.4 \%$. This agrees with the data reported in reference [27] quoting primary reference [28] for water, and has nearly tenfold improvement in relative error compared with reference [26].

\section{Conclusions}

This project demonstrates the capabilities of an entry-level FF-OCT device, which can be fabricated at very low cost in optics laboratories. Our instrument was not just an imaging tool, but also an accurate measurement tool. This instrument takes minutes for data acquisition as compared with fractions of a second for commercially available tools, and hence it is not suited for in-vivo biological observations where sample motion is a concern. Static ex-vivo biological as well as inorganic samples can be investigated with this device, and material characterization studies can be performed. We envisage the following improvements in a second iteration of our OCT instrument, which would improve performance significantly, without adding much to costs:

1. The use of a cooled monochrome CMOS camera available in Astronomy hobbyist stores for less than INR 16,000 (US\$250) could improve dynamic range and increase data capture speeds, as well as extend the response of the camera to the IR region.

2. Faster acquisition could let us acquire with shorter step sizes up to $39 \mathrm{~nm}$, opening up possibilities of phaseresolved imaging.

3. Using anti-reflection coated optics could reduce losses and allow operation in ambient light conditions.

4. GPU-assisted processing could improve processing speeds.

\section{Acknowledgements}

The authors wish to convey their gratitude to Bhagawan Sri Sathya Sai Baba, the founder Chancellor of their University, who guided and inspired us throughout this project. The authors' department has infrastructure funding from 
Department of Science and Technology, Government of India (DST) (FIST 2012-2017 SR/FST/PSI-172/2012).

\section{Compliance with ethical standards}

Conflict of interest The authors declare that they have no conflicts of interest to disclose.

\section{References}

[1] Puliafito C A, Hee M R, Schuman J S and Fujimoto J G 1996 Optical coherence tomography of ocular diseases. Slack Incorporated, USA, pp. 3-4

[2] Drexler W and Fujimoto J G (Eds.) 2008 Optical coherence tomography: technology and applications. Springer Science \& Business Media, Germany, pp. V-XXVIII, 24

[3] Swanson E A, Huang D, Lin C P, Puliafito C A, Hee M R and Fujimoto J G 1992 High-speed optical coherence domain reflectometry. Opt. Lett. 17(2): 151-153 https://doi.org/10. 1364/ol.17.000151.

[4] Fercher A F, Mengedoht K and Werner W 1998 Eye-length measurement by interferometry with partially coherent light. Opt. Lett. 13(3): 186-188. https://doi.org/10.1364/ol.13.000186.

[5] Hitzenberger C K, Drexler W and Fercher A F 1992 Measurement of corneal thickness by laser Doppler interferometry. Invest. Ophthalmol. Vis. Sci. 33(1): 98-103

[6] Fujimoto J and Swanson E 2016 The development, commercialization, and impact of Optical Coherence Tomography - history of optical coherence tomography Invest Ophthalmol Vis Sci. 57(9): OCT1-OCT13. https://doi.org/10. 1167/iovs.16-19963.

[7] Subhash H M, Hogan J N and Leahy M J 2015 Multiplereference optical coherence tomography for smartphone applications. SPIE Newsroom. https://doi.org/10.1117/2. 1201503.005807

[8] Duc Nguyen V, Weiss N, Beeker W, Hoekman M, Leinse A, Heideman R G, van Leeuwen T G and Kalkman J 2012 Integrated-optics-based swept-source optical coherence tomography. Opt. Lett. 37(23): 4820-4822. https://doi.org/ 10.1364/ol.37.004820.

[9] Beaurepaire E, Boccara A C, Lebec M, Blanchot L and SaintJalmes H 1998 Full-field optical coherence microscopy. Opt. Lett. 23(4): 244-246. https://doi.org/10.1364/ol.23.000244

[10] Dubois A, Vabre L, Boccara A C and Beaurepaire E 2002 High-resolution full-field optical coherence tomography with a Linnik microscope. Appl. Opt. 41(4): 805-812. https://doi. org/10.1364/ao.41.000805

[11] Subhash H M 2012 Full-field and single-shot full-field optical coherence tomography: a novel technique for biomedical imaging applications. Adv. Opt. Technol. https:// doi.org/10.1155/2012/435408

[12] Thouvenin O, Apelian C, Nahas A, Fink M and Boccara C 2017 Full-field optical coherence tomography as a diagnosis tool: recent progress with multimodal imaging. Appl. Sci. 7(3): 236. https://doi.org/10.3390/app7030236

[13] Huang D, Swanson E A, Lin C P, Schuman J S, Stinson W G, Chang W, Hee M R, Flotte T, Gregory K, Puliafito C A and Fujimoto J G 1991 Optical coherence tomography. Science 254(5035): 1178. https://doi.org/10.1126/science.1957169

[14] Fercher A F, Hitzenberger C K, Kamp G and El-Zaiat S Y 1995 Measurement of intraocular distances by backscattering spectral interferometry. Opt. Commun. 117(1-2): 43-48. https://doi.org/10.1016/0030-4018(95)00119-s

[15] Chinn S R, Swanson E A and Fujimoto J G 1997 Optical coherence tomography using a frequency-tunable optical source. Opt. Lett. 22(5): 340-342. https://doi.org/10.1364/ol. 22.000340

[16] Grebenyuk A, Federici A, Ryabukho V and Dubois A 2014 Numerically focused full-field swept-source optical coherence microscopy with low spatial coherence illumination. Appl. Opt. 53(8): 1697-1708. https://doi.org/10.1364/ao.53. 001697

[17] Canon, Inc. 2011 Canon EOS Rebel T3 EOS 1100 D instruction manual

[18] Johnson M K 2009 Capturing linear images. http://www.mit. $\mathrm{edu} / \sim \mathrm{kimo} / \mathrm{blog} / \mathrm{linear} . \mathrm{html}$

[19] Coffin D 2012 Decoding raw digital photos in Linux (version 9.27) [software]. http://www.cybercom.net/ dcoffin/dcraw/

[20] Grieve K, Dubois A, Simonutti M, Paques M, Sahel J, Le Gargasson J F and Boccara C 2005 In vivo anterior segment imaging in the rat eye with high speed white light full-field optical coherence tomography. Opt. Exp. 13(16): 6286-6295. https://doi.org/10.1364/opex.13.006286

[21] Eaton J W, Bateman D, Hauberg S and Wehbring R 2015 GNU Octave version 4.0. 0 manual: a high-level interactive language for numerical computations. http://www.gnu.org/ software/octave/doc/interpreter

[22] Kent B R 2015 3D scientific visualization with blender. Morgan \& Claypool, USA

[23] Blender Foundation 2016 Blender (version 2.77) [software]. http://www.blender.org/

[24] Wang L V and Wu H I 2012 Biomedical optics: principles and imaging. John Wiley \& Sons, USA, p. 208

[25] Coello Y, Xu B, Miller T L, Lozovoy V V and Dantus M 2007 Group-velocity dispersion measurements of water, seawater, and ocular components using multiphoton intrapulse interference phase scan. Appl. Opt. 46(35): 8394-8401. https://doi.org/10.1364/ao.46.008394

[26] Cheng H C and Liu Y C 2010 Simultaneous measurement of group refractive index and thickness of optical samples using optical coherence tomography. Appl. Opt. 49(5): 790-797. https://doi.org/10.1364/ao.49.000790

[27] Polyanskiy M N Refractive index database https://refracti veindex.info/ accessed on 2017-04-21

[28] Hale G M and Querry M R 1973 Optical constants of water in the $200-\mathrm{nm}$ to $200-\mu \mathrm{m}$ wavelength region. Appl. Opt. 12(3): 555-563. https://doi.org/10.1364/ao.12.000555 